\title{
EXAMPLES OF NONVANISHING CHERN-SIMONS INVARIANTS
}

\author{
JOHN J. MILLSON
}

\section{Introduction}

In this paper we study the Chern-Simons invariants. These invariants of $(4 n-1)$-dimensional Riemannian manifolds first appeared in Chern-Simons [1]. They are obstructions to conformal immersion of the Riemannian manifold in Euclidean space in much the same way as the Pontrjagin classes are to topological immersion. In Chern-Simons [1] a 3-dimensional example was given whose Chern-Simons invariant was nonzero. However, no higher-dimensional examples were given. Our first theorem gives a simple algebraic formula for these invariants for a spherical space form. In particular, for the Lens spaces $L\left(p ; q_{1}, q_{2}, \cdots, q_{2 n}\right)$ the invariants are expressible in terms of the elementary symmetric functions of $q_{1}, q_{2}, \cdots, q_{2 n}$ modulo $p$. Using this and judiciously choosing $p$ and the $q_{i}$ 's one can produce for each $n$, infinitely many Lens spaces $L\left(p ; q_{1}, q_{2}, \cdots, q_{2 n}\right)$ which immerse smoothly in $R^{4 n}$ but not conformally in $R^{4 n+2 n-2}$. This is the "best-possible" non-immersion result obtainable with the Chern-Simons invariants. For example, the 15 -dimsional Lens space $L(137 ; 1$, $10,100,41,136,127,37,96)$ immerses smoothly in $R^{16}$ but not conformally in $R^{22}$. As another application of our calculation for Lens spaces we give a residue formula for the Pontrjagin numbers of a $4 n$-manifold admitting a periodic diffeomorphism of prime order. We give here the formula for the case where the diffeomorphism $f$ has only isolated fixed points. Let $Q\left(p_{1}, p_{2}, \cdots, p_{n}\right)$ be a polynomial of the right weight in the Pontrjagin classes $p_{i}$ to obtain a Pontrjagin number $Q(M)$. Let $m_{1}, m_{2}, \cdots, m_{k}$ be the fixed points of $f$. If $p$ is the order of $f$, then $Q(M) \equiv \sum_{i=1}^{k} \operatorname{Res}\left(f, m_{i}\right)$, modulo $p$, where $\operatorname{Res}\left(f, m_{i}\right)$ is calculated as follows. Since $f$ leaves $m_{i}$ fixed, $d f$ maps the tangent space of $M$ at $m_{i}$ to itself. One can always (by averaging) assume $f$ preserves a metric on $M$, so $d f\left(m_{i}\right)$ is a rotation of order $p$. Let $\theta_{1}, \theta_{2}, \cdots, \theta_{2 n}$ be its rotation angles; that is, $d f\left(m_{i}\right)$ is similar to a block matrix :

Communicated by J. Simons, April 25, 1974. Submitted in partial fulfillment of the $\mathrm{Ph}$. D. requirements at the University of California, Berkeley. 


$$
\left(\begin{array}{rrrrr}
\cos \theta_{1} & -\sin \theta_{1} & & \multicolumn{2}{c}{0} \\
\sin \theta_{1} & \cos \theta_{1} & & & \\
& 0 & \ddots & & \\
& & & \cos \theta_{2 n} & -\sin \theta_{2 n} \\
& & & \sin \theta_{2 n} & \cos \theta_{2 n}
\end{array}\right) .
$$

Since $d f$ has order $p$ we have $\theta=2 \pi q / p$ for some integer $q$ which is determined modulo $p$. Then

$$
\operatorname{Res}\left(f, m_{i}\right)=-\frac{Q\left(\sigma_{1}\left(q_{1}^{2}, q_{2}^{2}, \cdots, q_{2 n}^{2}\right), \cdots, \sigma_{n}\left(q_{1}^{2}, q_{2}^{2}, \cdots, q_{2 n}^{2}\right)\right)}{q_{1} q_{2} \cdots q_{2 n}},
$$

where $\sigma_{i}$ is the $i$ th elementary symmetric function. This formula was also derived by Kosniowski [4] independently using a different method.

I would like to express my gratitude to James Simons, my thesis adviser, for explaining his ideas to me, for making many useful suggestions and most of all for showing me by example what is involved in doing mathematical research. I would also like to thank George Cooke who taught me algebraic topology and Madhav Nori who helped me with the number theory. Lastly I would like to thank the Canada Council for the Arts for its generous support during my years in graduate school.

\section{Review of definitions}

In Chern-Simons [1] the $T$ forms associated to a Riemannian manifold $M$ were defined. The form $T p_{n}$ is a $(4 n-1)$-form on the frame bundle of $M$ satisfying $d T p_{n}=\pi^{*} p_{n}$, where $\pi$ is the bundle projection and $p_{n}$ is the Pontrjagin form associated to the metric. In the case $p_{n}=0, T p_{n}$ defines a cohomology class on the frame bundle whose $R / Z$ reduction is the lift of a class from the base. In this way, we associate $R / Z$ cohomology classes to some Riemannian manifolds. These classes have the defect that there is no way to calculate them if $M$ is not parallelizable. The work of Cheeger-Simons [2] remedied this difficulty.

We will now give a review of the Simons $S$-characters. A more detailed treatment may be found in [1], [2]. They are the invariants of a geometric vector bundle, that is, a vector bundle with a connection. We will emphasize this notion throughout.

Let us begin by recalling the definition of these $S$-characters. We will not construct them in full generality but only in the Riemannian case. The universal object in Riemannian geometry is $B O(n)$ (or rather some large finite skeleton) equipped with the universal Levi-Civita connection on the canonical $n$-dimensional vector bundle over it. We obtain its curvature $\Omega$ and the Pontrjagin forms $p(\Omega)$ the natural globalization of $\Omega$. Now given any smooth $(4 k-1)$ - 
cycle $M$ in $G(n, N)$ (a finite approximation to $B O(n)$ ) either $M$ or $2 M$ bounds. If $M$ bounds, say $M=\partial W$ where $W$ is a smooth singular $4 k$ chain, define

$$
S p(M)=\overline{\int_{W} p(\Omega)}
$$

where - denotes reduction $\bmod Z$. That this number is independent of the choice of $W$ is clearly true. If $W_{1}$ is another choice with $\partial W_{1}=M$ then $W-W_{1}$ is a cycle; hence, $\int_{W-W_{1}} p(\Omega)=\int_{W} p(\Omega)-\int_{W_{1}} p(\Omega)$ is an integer. If $2 M$ bounds we have to be more careful. We choose an integral cochain $u$ which represents the integral Pontrjagin class and define

$$
S p(M)=\frac{1}{2}\left\{\overline{\int_{W} p(\Omega)-u(W)}\right\} .
$$

It is easily seen that $S p$ is independent of the choices of $W$ and $u . S p$ gives a homomorphism from the additive group of $(4 k-1)$-cycles to the circle, that is, it is a character of this group. Moreover, it is natural with respect to connection preserving bundle maps-as is easily seen from the definition.

Now given any Riemannian vector bundle (a vector bundle with a Riemannian metric and an invariant connection) $\pi: E \rightarrow M$, it is classified by a map to $B O(n)$ as a Riemannian bundle. That is, the connection on $E$ is the pullback connection from the universal connection in $B O(n)$. This follows from a theorem of Narasimhan and Ramanan [6]. Using this classifying map we can pull back the universal characters to $M$. Cheeger-Simons [2] showed that these characters depend only on the Riemannian bundle and not on the choice of classifying map.

An easy computation shows that in $B O(n), \delta S p=\overline{p(\Omega)}$ where $\delta$ is the $R / Z$ coboundary and $\overline{p(\Omega)}$ is the $R / Z$ cochain determined by the real Pontrjagin form. From this it follows that $S p$ defines a cohomology class on $M$ if and only if the Pontrjagin form $p(\Omega)$ is zero. Moreover, the above formula also implies that $S p$ lifts in the total space of the universal $O(n)$ bundle to an $R / Z$ primitive for $p$ (since this is already true on the base). Thus the lift of $S p$ and $T p$ are cohomologous as $R / Z$ cochains. In the case where $p(\Omega)$ vanishes, then the $R / Z$ class determined by $S p$ is the class whose lift is $T p$.

For a constant curvature manifold, all the Pontrjagin forms vanish. We thus have a host of $(4 k-1)$-dimensional cohomology classes associated with the Riemannian geometry of these manifolds. We now calculate these classes for constant positively-curved manifolds.

\section{The stability theorem}

Theorem. Given an n-manifold $M$ of constant positive curvature, there exists a trivial line bundle $L$ over $M$ so that $\tau(M) \oplus L$ admits a flat Riemannian connection compatible with the original connection on $\tau(M)$. Moreover, the $S$ 
classes of $\tau(M) \oplus L$ with this flat connection are the same as those of $\tau(M)$.

We say the connection $\bar{\nabla}$ on $\tau(M) \oplus L$ is compatible with the old connection $\nabla$ on $\tau(M)$ if given any section of $\tau(M) \oplus L$ of the form $(s, 0), s$ a section of $\tau(M)$, its derivative $\bar{\nabla}_{s}$ projected back into $\tau(M)$ coincides with $\nabla_{s}$.

Proof. Let $G$ be the fundamental group of $M^{n}$. Then $G$ is represented as a subgroup of $S O(n+1)$. One obtains the Riemannian tangent bundle of $M$ by quotienting the tangent bundle of $S^{n}$ by $G$. Now if $N$ denotes the normal field to $S^{n}$, then $\tau\left(S^{n}\right) \oplus N$ has the flat Euclidean connection. This connection and the orthogonal sum are preserved by $G$, and project down to $M$ to give the first statement of the theorem ( $L$ is the image of $N$ ).

Lemma A. The $S$ classes for the flat connection $\bar{\theta}$ on $\tau(M) \oplus L$ are the same as those for the Whitney sum connection $\theta \oplus \eta$ on $\tau(M) \oplus L$ where $\eta$ is the zero form as it takes values in $S O(1)$.

Proof. Join the two connections by a linear family of connections $(1-t) \theta$ $+t \bar{\theta}=\theta_{t}$. Then the variational formula from Cheeger-Simons [2] gives us

$$
S p(\bar{\theta})-S p(\theta)=\int_{0}^{1} p\left(\theta_{t}^{\prime}, \Omega_{t}, \cdots, \Omega_{t}\right) d t
$$

where $\theta_{t}^{\prime}=d \theta_{t} / d t$. We will show that the integrand is identically zero. Now $p$ is a polynomial in terms of the type $\theta_{t}^{\prime} \wedge \Omega_{t}^{l-1}$, since any invariant polynomial of degree $m$ for $0(n)$ is expressible as a polynomial in terms of the type trace $X^{l}, l \leq m$. We will show all such terms are zero. Our computations will be made on the principal bundle $F$ of frames in $\tau(M) \oplus L$. This bundle contains the subbundle $\tilde{F}$ of split frames $\left\{m, e_{1}, e_{2}, \cdots, e_{n}, N\right\}$ so that $e_{1}, e_{2}, \cdots, e_{n}$ is a frame for $\tau(M, m)$. If we can prove that $p\left(\theta_{t}^{\prime}, \Omega_{t}, \cdots, \Omega_{t}\right) \equiv 0$ on this subbundle, then the result will follow because $p$ is equivariant.

First note that restricted to $\tilde{F}, \theta$ has the form

$$
\left(\begin{array}{c|c}
A & 0 \\
\hline 0 & 0
\end{array}\right) \text {. }
$$

We say such a matrix is of type $\mathfrak{f} . \bar{\theta}$ has the form

$$
\left(\frac{A}{-p} \mid \frac{p}{}\right)
$$

where $p$ is a $1 \times n$ matrix. Thus $\theta_{t}^{\prime}=\left(\frac{0}{-p} \mid \frac{p}{0}\right)$. We say matrices of the form of $\theta_{t}^{\prime}$ have type $\mathfrak{p}$. Now let $\alpha=\bar{\theta}-\theta=\theta_{t}^{\prime}$. Then we have

$$
\begin{aligned}
\theta_{t} & =\theta+t \alpha, \\
\Omega_{t} & =d \theta_{i}+\frac{1}{2}\left[\theta_{t}, \theta_{t}\right]=d \theta+t d \alpha+\frac{1}{2}[\theta+t \alpha, \theta+t \alpha] \\
& =d \theta+\frac{1}{2}[\theta, \theta]+t\{d \alpha+[\theta, \alpha]\}+\frac{t^{2}}{2}[\alpha, \alpha] .
\end{aligned}
$$


Codazzi equation: $d \alpha+[\theta, \alpha]=0$.

Proof. $\bar{\theta}$ is a flat connection; hence

$$
0=\bar{\Omega}=d \bar{\theta}+\frac{1}{2}[\bar{\theta}, \bar{\theta}] .
$$

Writing $\bar{\theta}=\theta+\alpha$ (a direct sum $\mathfrak{f} \oplus \mathfrak{p}$ ) we obtain the direct sum splitting of $\bar{\Omega}$

$$
\begin{aligned}
\bar{\Omega}=d \bar{\theta}+\frac{1}{2}[\bar{\theta}, \bar{\theta}] & =d \theta+d \alpha+\frac{1}{2}[\theta+\alpha, \theta+\alpha] \\
& =d \theta+\frac{1}{2}[\theta, \theta]+\frac{1}{2}[\alpha, \alpha]+d \alpha+[\theta, \alpha]
\end{aligned}
$$

Setting the $\mathfrak{p}$ component of $\bar{\Omega}$ equal to zero gives

$$
d \alpha+[\theta, \alpha]=0 \text {. }
$$

Returning to the formula for $\Omega_{t}$, setting $d \alpha+[\theta, \alpha]=0$, and noting $[\alpha, \alpha]$ is of type $k$, we see $\Omega_{t}$ is of type $\mathfrak{f}$, and hence $\Omega_{t}^{l-1}$ is also of type $\mathfrak{f}$. But this means $\theta_{t}^{\prime} \wedge \Omega_{t}^{l-1}$ is a matrix of type $\mathfrak{p}$ (it is a product of a matrix of type $\mathfrak{f}$ with a matrix of type $\mathfrak{p}$ ); hence its trace vanishes, that is,

$$
\text { trace } \theta_{t}^{\prime} \wedge \Omega_{t}^{l-1}=0 \text {. }
$$

We have shown that the $S$-characters for $\tau(M) \oplus L$ equipped with the Euclidean connection are the same as for $\tau(M) \oplus L$ with the Whitney sum connection. Now $L$ is a trivial Riemannian line bundle. It is clear from the definition of the $S$ classes that $\tau(M) \oplus L$ with the Whitney sum connection and $\tau(M)$ have the same invariants.

Remark. This theorem is a special case of the Whitney-sum theorem of Cheeger-Simons [2]. We arrived at our theorem independently.

\section{Calculation of the Simons invariants for spherical space forms}

We now compute the invariants for the flat bundle $\tau(M) \oplus L$ in terms of the characteristic classes of the holonomy representation $\rho$. For more on the characteristic classes of group representations see Atiyah [1].

The representation $\rho: G \rightarrow S O(n+1)$ induces a map $B_{\rho}: B G \rightarrow B S O(n+1)$. There is a $C W$ decomposition of $B G$ so that $M$ is the $n$-skeleton; indeed, we have skeletal maps

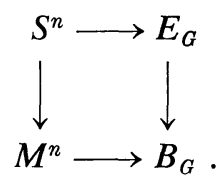

Proposition. The classifying map for $\tau(M) \oplus L$ is $B_{\rho} \circ i$.

Proof. Observe that $\tau(M) \oplus L=S^{n} \times{ }_{\rho} R^{n+1}$. 
Theorem. The $S$ invariants of $\tau(M)$ are given by

$$
S p_{i}(M)=-i^{*} \beta^{-1} p_{i}(\rho),
$$

where $p_{i}(\rho)$, the ith Pontrjagin class of the representation $\rho$, is given by $p_{i}(\rho)$ $=\left(B_{\rho}\right)^{*} p_{i}$, and $\beta$ is the $S^{1}$ Bockstein homomorphism.

Proof. Let $K$ be an $N$-dimensional skeleten of $B G, N \geq n+2$, obtained by attaching cells to $M^{n}$. If $\psi=B_{\rho} \mid K$, then $\psi \circ i$ still classifies $\tau(M) \oplus L$.

It is clear from their definition that the inverse Bockstein of an $S$ class is the negative of the corresponding integral Pontrjagin class. Since the Bockstein $\beta: H^{i}\left(K, S^{1}\right) \rightarrow H^{i+1}(K, Z)$ is an isomorphism for $0 \leq i \leq n$, the $S$-classes of the bundle over $K$ are just $-\beta^{-1} p_{i}(\rho)$. The theorem follows by naturality.

Remark. Since $B G$ is formed from $M$ by attaching cells of dimension greater than $n, i$ induces an onto map in $n$-dimensional integral homology and isomorphisms in lower dimensions. Thus $i$ is injective on $S^{1}$ cohomology of dimension $n$ and an isomorphism for dimensions less than $n$. Since $\beta^{-1}$ is an isomorphism we have

Corollary. $S p_{i}(M)$ vanishes if and only if $p_{i}(\rho)$ does for $4 i-1 \leq \operatorname{dim} M$.

Recall the definition of the Lens space $L\left(p ; q_{1}, \cdots, q_{n}\right) . U(n)$ acts on $S^{2 n-1} \subset C^{n} . Z_{p}$ is represented in $U(n)$ by $\rho(1)=\left(\begin{array}{lll}\lambda^{q_{1}} & & \\ & \ddots & \\ & \ddots & \\ 0 & & \lambda^{q_{n}}\end{array}\right)$ where $\lambda=e^{2 \pi i / p}$. The quotient of $S^{2 n-1}$ by this subgroup of $U(n)$ is the Lens space $L\left(p ; q_{1}, \cdots, q_{n}\right)$. By convention we assume $p$ and $q$ are relatively prime and the $q$ 's have no common factor. We assume $p$ is odd for convenience. We denote by $q_{i}^{\prime}$ the integer between 0 and $p$ which represents the multiplicative inverse of $q_{i}$ modulo $p$.

Given $\left(q_{1}, q_{2}, \cdots, q_{n}\right)$ we construct a model of $B Z_{p}$ (which is the one we will use from now on) whose $(2 n-1)$-skeleton is $L\left(p ; q_{1}, q_{2}, \cdots, q_{n}\right)$. First recall the definition of the infinite sphere $S^{\infty}$ :

$$
S^{\infty}=\left\{\left(z_{1}, z_{2}, \cdots\right): z_{i} \in C, \text { almost all } z_{i}=0 \text { and } \sum_{i=1}^{\infty}\left|z_{i}\right|^{2}=1\right\} .
$$

Choosing a generator $t$ of $Z_{p}$ we let $t$ act on $S^{\infty}$ by

$$
t \cdot\left(z_{1}, z_{2}, \cdots, z_{n}, z_{n+1}, \cdots\right)=\left(\lambda^{q_{1}} z_{1}, \lambda^{q_{2}} z_{2}, \cdots, \lambda^{q_{n}} z_{n}, \lambda z_{n+1}, \cdots\right) .
$$

The quotient space is the desired model. By representing $t$ in $U(1)$ as $e^{2 \pi i / p}$ we obtain a bundle over $B Z_{p}$ which we will call $H$. The Chern class of $H$ we will call the canonical generator of $H^{2}\left(B Z_{p}, Z\right.$ ) (adapted to the $q_{i}$ 's) and will label $x_{2}$.

Proposition. The Pontrjagin classes of $\rho$ are given by

$$
p_{i}(\rho)=\sigma_{i}\left(q_{1}^{2}, \cdots, q_{n}^{2}\right) x_{2}^{i},
$$

where $\sigma_{i}$ is the ith elementary symmetric function. 
Proof. Note that $p$ is already complex and diagonal. The bundle over $B Z_{p}\left(q_{1}, \cdots, q_{n}\right)$ is easily seen to be $H^{q_{1}} \oplus H^{q_{2}} \oplus \cdots \oplus H^{q_{n}}$. By definition $c(H)=1+x_{2}$. Hence $c\left(H^{q_{i}}\right)=1+q_{i} x_{2}$ and $p_{1}\left(H^{q_{i}}\right)=c_{1}\left(H^{q_{i}} \oplus C\right)=$ $c_{1}\left(H^{q_{i}} \oplus \bar{H}^{q_{i}}\right)=1-q_{i}^{2} x_{2}$.

The result follows from the Whitney sum formula (there is, of course, no 2-torsion in the cohomology of $B Z_{p}, p$ odd).

For later use we will need the $S$-numbers of $L\left(p, q_{1}, \cdots, q_{n}\right)$. For this we need a $(4 n-1)$-dimensional manifold, that is, an even number of $q_{i}$ 's.

We now compute the $S$-number corresponding to the top Pontrjagin class $S p_{n}\left(L\left(p ; q_{1}, \cdots, q_{2 n}\right)\right.$. Computations of the other numbers follow easily from this as will be seen.

Over $L\left(p ; q_{1}, \cdots, q_{2 n}\right)$ we have the Hopf bundle $H$, the restriction of the bundle $H$ over $B Z_{p}$. Alternatively it is the line bundle associated to the principal bundle $Z_{p} \rightarrow S^{4 n-1} \rightarrow L\left(p ; q_{1}, \cdots, q_{2 n}\right)$ by using the representation which sends the generator of $Z_{p}$ to $\lambda=e^{2 \pi i / p}$. Thus the Chern-class of $H$ is the restriction of $x_{2}$, the canonical generator of $H^{2}\left(B Z_{p}, Z\right)$, to $L\left(p ; q_{1}, \cdots, q_{2 n}\right)$. This restriction we will also call $x_{2}$. Now we have seen that the calculation of $S p_{n}$ for the tangent bundle of $L\left(p ; q_{1}, \cdots, q_{2 n}\right)$ which we denote $S p_{n}\left(L\left(p ; q_{1}, \cdots, q_{2 n}\right)\right)$ is reduced to calculating:

$$
\begin{aligned}
& \left\langle\sigma_{n}\left(q_{1}^{2}, q_{2}^{2}, \cdots, q_{2 n}^{2}\right) \beta^{-1} x_{2} \cup x_{2}^{2 n-1}, L\left(p ; q_{1}, \cdots, q_{2 n}\right)\right\rangle \\
& \quad=\sigma_{n}\left(q_{1}^{2}, q_{2}^{2}, \cdots, q_{2 n}^{2}\right)\left\langle\beta^{-1} x_{2} \cup x_{2}^{2 n-1} ; L\left(p ; q_{1}, \cdots, q_{2 n}\right)\right\rangle .
\end{aligned}
$$

But the quantity inside the brackets is merely $S p_{n}$ for the bundle $H \oplus H \oplus$ $\cdots \oplus H$ (taken $2 n$ times) over $L\left(p ; q_{1}, \cdots, q_{2 n}\right)$.

We simplify the calculation still further by noting that there is a degree $q_{1}^{\prime} q_{2}^{\prime} \cdots q_{2 n}^{\prime}$ map from $L\left(p ; q_{1}, \cdots, q_{2 n}\right)$ to $L(p ; 1,1, \cdots, 1)$ obtained from $\left(z_{1}, z_{2}, \cdots, z_{2 n}\right) \stackrel{\varphi}{\longrightarrow}\left(z_{1}^{q_{1}^{\prime}}, z_{2}^{q_{2}^{\prime}}, \cdots, z_{2 n}^{q_{2 n}^{\prime}}\right)$. Moreover, $\varphi$ can clearly be covered by a bundle map from the bundle $H$ over $L\left(p ; q_{1}, \cdots, q_{2 n}\right)$ to the bundle $H$ over $L(p ; 1,1, \cdots, 1)$. Thus $\varphi^{*} x_{2}=x_{2}$, and we obtain

$$
\begin{aligned}
\left\langle\beta^{-1} x_{2}\right. & \left.\cup x_{2}^{2 n-1}, L(p ; 1,1, \cdots, 1)\right\rangle \\
& =\frac{1}{q_{1}^{\prime} q_{2}^{\prime}, \cdots, q_{2 n}^{\prime}}\left\langle\beta^{-1} x_{2} \cup x_{2}^{2 n-1}, \varphi_{*} L\left(p ; q_{1}, \cdots, q_{2 n}\right)\right\rangle \\
& =\frac{1}{q_{1}^{\prime} q_{2}^{\prime}, \cdots, q_{2 n}^{\prime}}\left\langle\beta^{-1} \varphi^{*} x_{2} \cup \varphi^{*} x_{2}^{2 n-1}, L\left(p ; q_{1}, \cdots, q_{2 n}\right)\right\rangle \\
& =\frac{1}{q_{1}^{\prime} q_{2}^{\prime}, \cdots, q_{2 n}^{\prime}}\left\langle\beta^{-1} x_{2} \cup x_{2}^{2 n-1}, L\left(p ; q_{1}, \cdots, q_{2 n}\right)\right\rangle .
\end{aligned}
$$

We then see that

$$
\begin{aligned}
& \operatorname{Sp}_{n}\left(L\left(p ; q_{1}, \cdots, q_{2 n}\right)\right) \\
& \quad=-q_{1}^{\prime} q_{2}^{\prime} \cdots q_{2 n}^{\prime} \sigma_{n}\left(q_{1}^{2}, \cdots, q_{2 n}^{2}\right)\left\langle\beta^{-1} x_{2} \cup x_{2}^{2 n-1}, L(p ; 1, \cdots, 1)\right\rangle,
\end{aligned}
$$


where $q_{i}^{\prime}$ is an integer whose residue $\bmod p$ is the multiplicative inverse of that of $q_{i} \bmod p$.

To evaluate the quantity in parentheses we can use several different methods.

The quantity $\left\langle\beta^{-1} x_{2} \cup x_{2}^{2 n-1}, L(p ; 1,1, \cdots, 1)\right\rangle$ is just the linking number of the Poincaré duals of $x_{2}$ and $x_{2}^{2 n-1}$ (see for example Seifert and Threlfall, Lehrbuch der Topologie, pp. 277-280). Let $\left[z_{1}, \cdots, z_{2 n}\right], z_{i} \in C$ and $\left|z_{1}\right|^{2}+$ $\left|z_{2}\right|^{2}+\cdots+\left|z_{2 n}\right|^{2}=1$ be the "homogeneous coordinates" in the Lens space $L(p ; 1,1, \cdots, 1)$; that is, $\left[z_{1}, z_{2}, \cdots, z_{2 n}\right]$ is the equivalence class of $\left(z_{1}, z_{2}\right.$, $\left.\cdots, z_{2 n}\right) \in C^{2 n}$ under the diagonal $Z_{p}$ action, then the Poincare dual of $x_{2}$ is represented by the sub-Lens space $\left\{\left[z_{1}, z_{2}, \cdots, z_{2 n-1}, 0\right]\right\}=L(p ; 1,1, \cdots, 1)$ where this time there are $(2 n-1) 1$ 's. The Poincare dual of $x_{2}^{2 n-1}$ is the subLens-space (actually a circle) $\left[0,0, \cdots, 0, z_{2 n}\right] . p$ times this later manifold bounds the singular disk $\left\{\left[0,0, \cdots, \cos \frac{\pi t}{2}, \sin \frac{\pi t}{2} z_{2 n}\right],\left|z_{2 n}\right|=1,0 \leq t \leq 1\right\}$, Since the Poincaré dual of $x_{2}$ intersects this disk at one point we find $1 / p$ for the desired linking number.

Finally, then we obtain

$$
\begin{aligned}
S p_{n}\left(L\left(p ; q_{1}, q_{2}, \cdots, q_{2 n}\right)\right) & \equiv-\frac{\sigma_{n}\left(q_{1}^{2}, \cdots, q_{2 n}^{2}\right)}{p} q_{1}^{\prime} q_{2}^{\prime} \cdots q_{2 n}^{\prime} \quad \bmod Z \\
& \equiv-\frac{\sigma_{n}\left(q_{1}^{2}, \cdots, q_{2 n}^{2}\right)}{q_{1} q_{2} \cdots q_{2 n}} \bmod p
\end{aligned}
$$

Note the previous proof also gives that: for any polynomial $Q\left(t_{1}, t_{2}, \cdots, t_{n}\right)$ of weight $n$ (i.e., for each monomial $t_{1}^{a_{1}} t_{2}^{a_{2}} \cdots t_{n}^{a_{n}}$ occurring in $Q$ we have $\left.a_{1}+2 a_{2}+\cdots+n a_{n}=n\right)$,

$$
S Q\left(p_{1}, p_{2}, \cdots, p_{n}\right) \equiv-\frac{Q\left(\sigma_{1}\left(q_{1}^{2}, \cdots, q_{2 n}^{2}\right), \cdots, \sigma_{n}\left(q_{1}^{2}, \cdots, q_{2 n}^{2}\right)\right)}{q_{1} q_{2} \cdots q_{2 n}} \quad \bmod p
$$

\section{Application to comformal immersions}

To construct the manifolds promised in the introduction we construct Lens spaces so that all $S$-classes but the highest are zero. Recall that for $L\left(p ; q_{1}\right.$, $\left.\cdots, q_{2 n}\right)$

$$
S p_{i}=0 \Longleftrightarrow \sigma_{i}\left(q_{1}^{2}, \cdots, q_{2 n}^{2}\right) \equiv 0 \quad \bmod p
$$

Also assume for convenience that $p$ is an odd prime.

Thus to construct a Lens space whose only nonvanishing $S$-class is the top class we must solve the following number theory problem. Given an even number $2 n$, find a prime $p$ and a $2 n$-tuple $\left\{q_{1}, q_{2}, \cdots, q_{2 n}\right\}$ so that for $1<n$ 


$$
\begin{array}{ll}
\sigma_{i}\left(q_{1}^{2}, q_{2}^{2}, \cdots, q_{2 n}^{2}\right) \equiv 0 & \bmod p, \\
\sigma_{n}\left(q_{1}^{2}, q_{2}^{2}, \cdots, q_{2 n}^{2}\right) \not \equiv 0 & \bmod p .
\end{array}
$$

We now find a sufficient condition which the $q_{i}$ 's themselves must satisfy in order that the $q_{i}^{2}$ 's satisfy the above equation. Suppose we have solved the equations

$$
\begin{aligned}
\sigma_{i}\left(q_{1}, \cdots, q_{2 n}\right) \equiv 0 \quad & \bmod p, 1 \leq i<2 n, \\
& \text { with none of the } q_{i} \text { 's zero } \bmod p .
\end{aligned}
$$

$\sigma_{i}\left(q_{1}^{2}, \cdots, q_{2 n}^{2}\right)$ is a symmetric function of the $q_{i}$ 's and therefore a polynomial in $\sigma_{k}\left(q_{1}, \cdots, q_{2 n}\right)$. Now, if $1<n$ we must have $k<2 n$. Hence for the $q_{i}$ 's which are solutions of ${ }^{*}$ we have $\sigma_{i}\left(q_{1}^{2}, \cdots, q_{2 n}^{2}\right) \equiv 0, \bmod p$ for $i<n$. Now the expansion of $\sigma_{n}\left(q_{1}^{2}, \cdots, q_{2 n}^{2}\right)$, the only term of which does not give zero when evaluated at our special $q_{i}$ 's, is $\sigma_{2 n}\left(q_{1}, \cdots, q_{2 n}\right)$. This appears multiplied by a universal constant which we evaluate

$$
\sigma_{n}\left(X_{1}^{2}, \cdots, X_{2 n}^{2}\right)=C \sigma_{2 n}\left(X_{1}, \cdots, X_{2 n}\right) \bmod \text { lower terms. }
$$

To evaluate $C$ we choose $X_{1}=1, X_{2}=\xi, \cdots, X_{2 n}=\xi^{2 n-1}$ where $\xi$ is a primitive $(2 n)$ th root of unity. $\sigma_{2 n}\left(1, \xi, \cdots, \xi^{2 n-1}\right)=1$. On the other hand, $\sigma_{n}\left(1, \xi^{2}, \cdots, \xi^{4 n-2}\right)=(-1)^{n+1} 2$, as one sees easily.

Since $p$ is odd, $\sigma_{2 n}\left(q_{1}, \cdots, q_{2 n}\right)=q_{1} \cdots q_{2 n} \not \equiv 0, \bmod p \Rightarrow \sigma_{n}\left(q_{1}^{2}, \cdots, q_{2 n}^{2}\right) \not \equiv$ $0, \bmod p$. From this we see that it is enough to find $q_{1}, \cdots, q_{2 n}$ which satisfy $*$. In order that $*$ be satisfied it is sufficient that

$$
\left(X-q_{1}\right)\left(X-q_{2}\right) \cdots\left(X-q_{2 n}\right) \equiv X^{2 n}-1 \quad \bmod p,
$$

that is, the polynomial $X^{2 n}-1$ splits completely over the field $Z_{p}$. For this it is enough that $Z_{p}$ contain a primitive $(2 n)$ th root of unity, that is, $Z_{p}^{*}$ have an element of precisely $2 n$. Since $Z_{p}^{*}$ is cyclic (recall $p$ is prime) this is equivalent to $2 n \mid(p-1)$, that is, $p=2 n k+1$. The existence of an infinite number of primes of this form is guaranteed by the Dirichlet prime theorem. An example is $2 n=8, p=137, \xi=10$, for which the resulting Lens space is

$$
L(137 ; 1,10,100,41,136,127,37,96) \text {. }
$$

Now recall that modulo 2 torsion the normal Pontrjagin classes of a bundle $E$ are defined recursively by:

$$
p_{i}^{\perp}=-p_{i}-p_{i-1} p_{1}^{\perp}-\cdots-p_{i-1}^{\perp} p_{1} .
$$

Whitney duality theorem tells us that the classes $p_{i}^{\frac{1}{i}}$ are precisely the Pontrjagin classes of the stable inverse bundle $E^{\perp}$. Cheeger-Simons [2] have shown that 
precisely the same situation holds for the $S$-classes, that is, the $S$-classes of the Riemannian inverse bundle $E^{\perp}$ are just the $S$-classes obtained from the original bundle $E$ by applying the $S$-construction to the pair $\left(p_{1}^{\perp}(\Omega), u_{i}^{\perp}\right)$ where $u_{i}^{\perp}$ is an integer cocycle representing $p_{i}^{\perp}$.

Thus, is $S p_{m}^{\perp}(E)$ does not vanish, then $E$ does not admit a Riemannian inverse bundle of dimension less than $2 \mathrm{~m}$. In the case where $E$ is the tangent bundle of a Riemannian manifold, this gives a lower bound on the codimension of an isometric or conformal immersion.

Now for Lens spaces of dimension $4 n-1$ all the $S$-classes except for $S p_{n}$ are just minus the inverse images of the corresponding integral Pontrjagin classes under the Bockstein homomorphism. For the Lens spaces we have just constructed $S p_{i}^{\perp}=0, i \neq n$, and $S p_{n}^{\perp} \neq 0$. Indeed, $S p_{i}^{\perp}=\beta^{-1}\left(p_{i}^{\perp}\right)=\beta^{-1}\left(p_{i}\right)$ $+\beta^{-1}(Q)$, where $Q$ is a polynomial in $p_{j}^{\frac{1}{j}}, p_{j}, j<i$, which we can assume is zero by induction.

It follows then that these Lens spaces do not immerse isometrically in codimension $2 n-1$. For $L(137 ; 1,10,100,41,136,127,37,96), S p_{4}^{\perp} \neq 0$, hence it does not immerse isometrically in codimension 7. Since this Lens space is 15 dimensional, it does not immerse isometrically in $R^{22}$.

We still must show that we can find special Lens spaces which satisfy* and immerse smoothly in codimension 1. By standard immersion theory it is enough to construct special Lens spaces with stably-trivial tangent bundles. That this can be done follows immediately from the following fundamental lemma of Kervaire [4] :

Let $\tau$ be a stable $S O(m)$ bundle over a complex $K$ (i.e., $\operatorname{dim} K<m$ ), and $S$ a cross-section of $\tau \mid K^{4 l-1}$. Then the obstruction $O_{l}(\tau, S) \in H^{4 l}\left(K, \pi_{4 l-1}(S O(m))\right)$ is related to the Pontrjagin class $p_{l}(\tau)$ by

$$
p_{l}(\tau)=a_{l}(2 l-1) ! 0_{4 l}(\tau, S),
$$

where $a_{l}=2, l$ odd; $a_{l}=1, l$ even. Since the special Lens spaces which we have constructed have the property $p_{l}=0$ for all $l$, by choosing the prime $p$ sufficiently large (so that $p>(2 l-1)$ ! for all possible $l$ ) we can ensure

$$
0_{l}(\tau, S)=0 \quad \text { for all } l \text {. }
$$

If the Lens space under consideration has dimension $4 n-1$, then the last obstruction occurs in dimension $4 n-4$ so that $l=n-1$. Thus $p>(2 n-3)$ ! will guarantee all obstructions to stable trivialization vanish.

Note that $137>(8-3) !=5 !=120$. Hence $L(137 ; 1,10,100,41,136$, $127,37,96)$ immerses smoothly in $R^{16}$.

In summary, for each $n$ we have constructed infinitely many Lens spaces of dimension $4 n-1$, immersing smoothly in $R^{4 n}$ but not conformally in $R^{4 n+2 n-2}$. 


\section{5. $Z_{p}$ actions on $4 k$-manifolds and characteristic numbers, and the residue formula for isolated fixed points}

Suppose an oriented $4 n$-manifold $M$ admits a periodic diffeomorphism $f$ with isolated fixed points $m_{1}, m_{2}, \cdots, m_{k}$. Choose a metric on $M$ so that $f$ is an isometry for that metric. $d f$ maps the tangent space at the fixed point to itself and, relative to some basis, may be written in rotation blocks :

$$
\left[\begin{array}{cc}
\cos \frac{2 \pi q_{1}}{p} & -\sin \frac{2 \pi q_{1}}{p} \\
\sin \frac{2 \pi q_{1}}{p} & \cos \frac{2 \pi q_{1}}{p} \\
\hline & 0
\end{array} \mid=\right.
$$

For each fixed point $m_{i}$ we get $2 n$ rotation angles $2 \pi q_{1} / p, 2 \pi q_{2} / p, \cdots, 2 \pi q_{2 n} / p$. Now let $Q\left(t_{1}, \cdots, t_{k}\right)$ be a polynomial of weight $n$. Then corresponding to $Q$ there is a Pontrjagin number $\int_{M} Q\left(p_{1}, \cdots, p_{k}\right)=Q([M])$ where $p_{i}$ denotes the $i$ th Pontrjagin class, and our theorem implies

$$
Q([M]) \equiv-\sum_{\text {fixed points }} \frac{Q\left(\sigma_{1}\left(q_{1}^{2}, \cdots, q_{2 n}^{2}\right), \cdots, \sigma_{k}\left(q_{1}^{2}, \cdots, q_{2 n}^{2}\right)\right)}{q_{1} q_{2} \cdots q_{2 n}} \quad \bmod p
$$

Proof. We replace the metric by a metric which is flat around each fixed point and for which $f$ acts isometrically. The procedure is the same as flattening a polar cap of the sphere to get a flat metric around the north pole. Formally, one proceeds as follows. Let $m_{0}$ be a fixed point and $U\left(m_{0}\right)$ a neighborhood so that the exponential map is a diffeomorphism from some open set in $T\left(M, m_{0}\right)$ onto $U\left(m_{0}\right)$. Define a new metric $(())$ in $U\left(m_{0}\right)$ as follows. If $V$ and $W$ are two tangent vectors at $m \in U\left(m_{0}\right),((V, W))=\left(\exp _{m_{0} *}^{-1} V, \exp _{m_{0} *}^{-1} W\right)$ where $($, denotes the inner product in $T\left(M, m_{0}\right)$. We interpolate between this metric and the original metric in some annulus around boundary $U_{0}$ using a function of the geodesic distance from the fixed point. Do this for all fixed points $m_{i}$. Since $f$ was an isometry of the original metric it commutes with the exponential map. From this it is easy to deduce that $f$ is an isometry of $(()$,$) . Now if \Omega$ is the curvature form of $(()$,$) , then$

$$
Q([M])=\int_{M} Q\left(p_{1}(\Omega), \cdots, p_{k}(\Omega)\right)=\int_{M-\underset{i=1}{i}\left\{m_{i}\right\}} Q\left(p_{1}(\Omega), \cdots, p_{k}(\Omega)\right)
$$

since the integral is not changed by removing a set of measure zero. If $B_{i}(r)$ is the ball of radius $r$ around the fixed point $m_{i}$, we obtain 


$$
Q([M])=\lim _{r \rightarrow 0} \int_{M-\underset{\substack{\cup \\ i=1}}{u} B_{i}(r)} Q\left(p_{1}(\Omega), \cdots, p_{k}(\Omega)\right) .
$$

Now, $M-\bigcup_{i=1}^{k} B_{i}(r)$ is a manifold with boundary a disjoint collection of $(4 n$ - 1)-spheres. Choose $r$ so small that each sphere $S_{i}^{4 n-1}$ is contained inside the neighborhood $U\left(m_{i}\right)$. Each sphere admits a fixed point free isometric $Z_{p}$ action obtained by restricting $f$. Taking the quotient of $M-\bigcup_{i=1}^{k} B_{i}(r)=W$ we obtain a manifold $\bar{W}$ with boundary a disjoint collection of Lens spaces $L_{i}\left(p ; q_{1}\right.$, $\left.\cdots, q_{2 n}\right)$. The $q_{i}$ 's are of course determined by the rotation angles of $d f$. Now $\int_{W} Q\left(p_{1}(\Omega), \cdots, p_{k}(\Omega)\right)=\frac{1}{p} \int_{\bar{W}} Q\left(p_{1}(\Omega), \cdots,\left(p_{i}(\Omega)\right) \equiv S Q\left(T(\bar{M}) \mid \bigcup_{i=1}^{k} L_{i}\right), \bmod \right.$

$Z$. By the construction of our metric, the bundle $T(\bar{M}) \mid L_{i}$ is just the locally flat Euclidean bundle of $\S 2$ whose $S$ invariants we calculated. The theorem follows by passing to the limit as $r \rightarrow 0$.

Remark. One can obtain a formula for smooth $Z_{p}$ actions with general fixed point sets by computing the $S$-characters of Lens space bundles in terms of the $S$-characters of the fiber and characteristic classes of the base, according to the Simons-Cheeger product formula. (See Cheeger-Simons [2].) Also, one can deduce congruences corresponding to polynomials of degree less than $4 k$. We will do neither of these and we refer the reader to the paper of Kosniowski [5] for these formulas.

We had wondered if the Atiyah-Hirzebruch theorem that a spin-manifold admitting any nontrivial $S^{1}$ action has vanishing $\hat{A}$ genus generalized to the $\bmod p$ case. However, Nigel Hitchin pointed out that the quartic surface $z_{0}^{4}+$ $z_{1}^{4}+z_{2}^{4}+z_{3}^{4}=0$ in $C P^{3}$ is spin, has $\hat{A}$ genus 2 , and admits a $Z_{3}$ action (in fact an $S_{4}$ action as permutations of the coordinates).

\section{References}

[1] M. F. Atiyah, Characters and cohomology of finite groups, Inst. Hautes Études Sci. Publ. Math. No. 9 (1961).

[2] J. Cheeger \& J. Simons, Differential characters and geometric invariants, to appear.

[ 3 ] S. Chern \& J. Simons, Some cohomology classes in principal fibre bundles and their application to Riemannian geometry, Proc. Nat. Acad. Sci. U.S.A. 68 (1971) 791-794.

[ 4 ] M. A. Kervaire, A note on obstructions and characteristic classes, Amer. J. Math. 81 (1959) 773-784.

[ 5 ] C. Kosniowski, What the fixed points say about a $\mathrm{Z} / \mathrm{p}$ manifold, J. London Math. Soc. 8 (1974) 179-186.

[ 6 ] M.S. Narasimhan \& S. Ramanan, Existence of universal connections, Amer. J. Math. 83 (1961) 563-572, 85 (1963) 223-231. 